\title{
Design of Microwave-based Brain Tumor Detection Framework with the Development of Sparse and Low-Rank Compressive Sensing Image Reconstruction
}

\author{
Hermawan Rahman Sholeh ${ }^{1}$, Mia Rizkinia ${ }^{1 *}$, Basari Basari ${ }^{2,3}$ \\ ${ }^{1}$ Department of Electrical Engineering, Faculty of Engineering, Universitas Indonesia, Kampus UI Depok, \\ Depok 16424, Indonesia \\ ${ }^{2}$ Biomedical Engineering, Department of Electrical Engineering, Faculty of Engineering, Universitas \\ Indonesia, Kampus UI Depok, Depok 16424, Indonesia \\ ${ }^{3}$ Research Center for Biomedical Engineering, Faculty of Engineering, Universitas Indonesia, Kampus UI \\ Depok, Depok 16424, Indonesia
}

\begin{abstract}
Cancer is one of the leading causes of death, and the brain is one of the body's cancerprone organs. The early detection of brain tumors can reduce cancer risk, which is practically assisted and conducted using scanners such as computed tomography (CT) and magnetic resonance imaging (MRI). However, those modalities are high-cost and large-sized, and they have a side effect risk to health. Alternatively, microwave imaging offers a novel cancer scanning method for early detection with low cost, small size and low health risk. Consequently, this research designs and creates a framework with a novel microwave image reconstruction algorithm inside. The framework is a component of the controller and image reconstructor for a portable microwavebased brain tumor detector that is open source and multi-platform. For the novel algorithm, this research proposes a CS-based imaging algorithm by exploiting the data's sparse and low-rank properties. The experiment shows that the proposed algorithm can give better qualitative and quantitative reconstruction results compared to a full-sampling-based as well as CS-based algorithm.
\end{abstract}

Keywords: Compressive sensing; Framework; Image reconstruction; Low-rank; Microwave imaging; Sparse

\section{Introduction}

In Cancer is the second-leading cause of death globally. According to the WHO, the death rate due to cancer reached 9.6 million in 2018 (WHO, 2018). The brain is one of the organs susceptible to cancer. The early detection of brain tumors is essential to mitigate the risk of cancer. There are many examples of tumor detection using imaging technologies such as X-ray, computed tomography (CT), magnetic resonance imaging (MRI) and positron emission tomography (PET) (Gao and Jiang, 2013). However, these modalities still have disadvantages such as radiation level, device complexity, operational costs and size (Chandra et al., 2015). Therefore, researchers develop a new modality by utilizing microwaves (Dilman et al., 2017; Shtoda et al., 2017).

Microwave imaging has used various algorithms to reconstruct the scan results, such

*Corresponding author's email: mia@ui.ac.id,, Tel.: +62-21-7270078; Fax: + 62-21-7270077 doi: 10.14716/ijtech.v11i5.4329 
as time-domain inversion (Ali and Moghaddam, 2010), ultra-wideband magnitude combined tomography (UPC), algebraic reconstruction technique (ART) (Elevani et al., 2016, Elevani et al.,2017), simultaneous algebraic reconstruction technique (Aprilliyani et al., 2017), filtered back projection (FBP) (Ramadhan et al., 2017; Ramdani et al., 2018) and the algorithm based on compressive sensing (CS) (Basari and Ramdani, 2019). However, UPC, ART and FBP algorithms have disadvantages because they require much data to get decent results. Meanwhile, the compressive sensing approach reduces the amount of data needed for reconstruction because it uses sampled data. The data also tend to be sparse in a frequency domain, that is having many elements containing zero value among the total data set (Donoho et al., 2016; Basari and Ramdani, 2019). Previous research proposed CS with total variation (TV) regularization solved by the alternating direct method of multipliers (ADMM) (Razzak et al., 2019). The method shows a smoother reconstructed image and lower mean square error (MSE) than SART and sparse CS.

The research contribution lies in the proposed image reconstruction algorithm for the software aspect and the proposed framework for the portable hardware aspect. The proposed algorithm improves image reconstruction results by using the low-rank property of the data. It combines sparse and low-rank properties of the data based on the compressive sensing approach. The idea is that the microwave measurement data are not only sparse but also low rank. The low-rank property is represented by imposing a nuclear norm (the sum of a matrix's singular value). It has been utilized for facial recognition (Luo et al., 2014), feature extraction (Yang et al., 2017), hyperspectral unmixing (Giampouras et al., 2016), CT image reconstruction (Yang et al., 2017) and MRI reconstruction (Ulas et al., 2016) due to its robustness. The data are said to be low rank because data matrices tend to be highly correlated. In an implementation, the nuclear norm is imposed on the CS optimization problem to consider low ranking.

Researchers have developed simple, low-cost and portable medical devices (Hugeng and Kurniawan, 2016). This research introduces the design of a framework that is universal, open-source and multi-platform concerning the hardware aspect. The framework is for a controller and for packaging the developed image reconstruction algorithm. It can run on a card-sized computer, such as the Raspberry Pi, to be used as a portable brain tumor detector component. The framework can be operated as if a user operates a portable brain tumor detector.

The rest of this paper is organised as follows. Section 2 describes the details of the proposed method in this paper. Section 3 shows parameter settings, results and our respective analyses. Finally, Section 4 concludes this study.

\section{Methods}

\subsection{Subject}

This research consists of two works. The first is designing and implementing a framework for the controller and image reconstruction of a portable microwave-based brain tumor detector. The second work is developing a microwave-imaging algorithm using sparse and low-rank compressive sensing (SLR-CS).

\subsection{Design and Implementation of Framework}

\subsubsection{Hardware configuration}

Microwave system imaging (MSI) hardware generally consists of a microwave signal transmitter and receivers such as a vector network analyzer (VNA), antenna arrays, radio frequency switches for switching between antennas (Chandra et al., 2015) and computers 
for controlling the hardware (VNA, switch, and antenna), data acquisition and image reconstruction.

Hugeng and Kurniawan (2016) configured their hardware consisting of Arduino Uno to detect and acquire a signal and PC for signal analysis and classification using an expert system. In this research, the framework is designed to run on a hardware platform, as shown in Figure 1a. The computer used is the Raspberry Pi 3B + with a five-inch display touchscreen. The connection with devices uses the USB port and GPIO port. Raspberry Pi was chosen for two reasons. The first is the purchase price. The second is that it can fulfill the functions needed as a controller and image reconstruction component of a portable microwave-based brain tumor detector. The Raspberry Pi is a card-sized computer. It has the required features to run the framework, such as mathematical computing with Python, GPIO and an interface. The interface is used to give commands to microwave transmitting and receiving devices such as VNA via a USB port using a serial module or LabView (Raspberry Pi Foundation, 2020; Copper Mountain Technologies, 2020; Ranson, 2015).

\subsubsection{Framework configuration}

The framework is designed so that after the user puts the antenna sensor to the patient's head, the user only clicks a "Run" button. The framework system will automatically do the measurement, data acquisition and image reconstruction, as well as display an image. Figure $1 \mathrm{~b}$ shows the design of modules forming the framework. The modules consist of three parts, namely:

1. GUI. That is, a module for managing the display, for example operating buttons, displaying reconstruction results and displaying the system's work processes.

2. Controller. That is, a module for controlling the process and device (LCD, VNA and Switch). This module consists of the following sub-modules:

a) Process Controller. That is, as a controller for the stages of the detection process, starting from data acquisition to displaying the image reconstruction results.

b) Measurement Controller. That is, to manage the measurement and data acquisition process by controlling the VNA and switch.

c) Reconstructor. That is, a module that contains an image reconstruction algorithm.

d) Hardware Interface. That is, a module as an interface between the three modules above and the hardware (VNA and Switch).

A GUI to operate the portable microwave-based brain tumor detector is shown in Figure 4(b). The GUI on the framework has four buttons and a text box to display the system activity log. The button consists of "Run" to run the image detection and reconstruction process, "Settings" to set system parameters, "Clear" to clear the activity log and "Quit" to exit the application. When the "Run" button is pressed, the framework performs the devices' (Switch, VNA) initialization, measurement and data acquisition, and image reconstruction, and displays the image reconstruction results.

We simulated giving orders and taking measurement results using the Arduino Uno to carry out the data acquisition process. We use the serial communication feature on the Arduino so the framework can simulate giving data acquisition commands to VNA and Switch devices. Subsequently, the device responds by sending measurement data. Therefore, in this research, we program the Arduino by writing the command code and measurement data to be sent.

\subsubsection{Framework implementation and evaluation}

The framework is written in Python. Python was chosen due to its features, for example support OOP. It is easy to develop, open-source and multi-platform, and it has many 
libraries for scientific computation and device control. In this research, the code was divided into four files: the GUI file for the display; the algorithm file for an image reconstruction algorithm, the development of which is explained in Section 2.2; the main file as a hardware controller and workflow controller; and the interface file as a liaison between the framework and hardware (VNA, Switch).

It conducts two types of testing: framework functionality and image reconstruction. Functionality testing checks how the framework operates based on the determined specifications. Meanwhile, reconstruction testing is explained in Section 2.2.2.

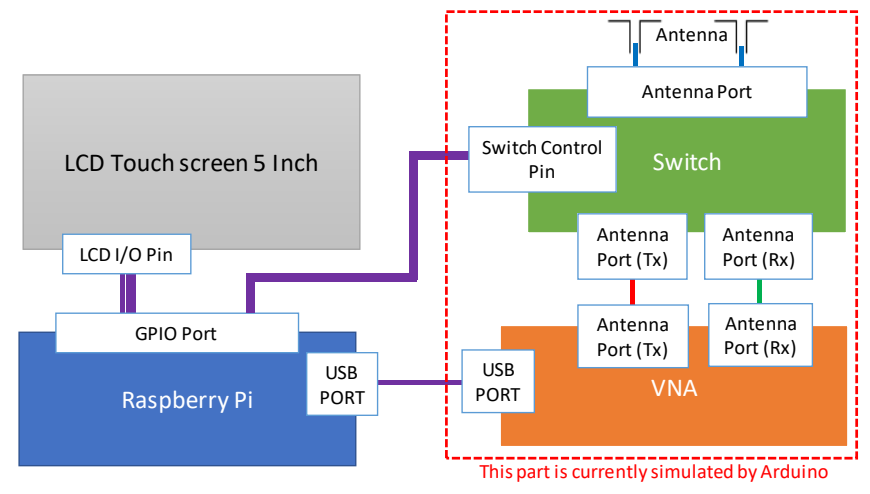

(a)

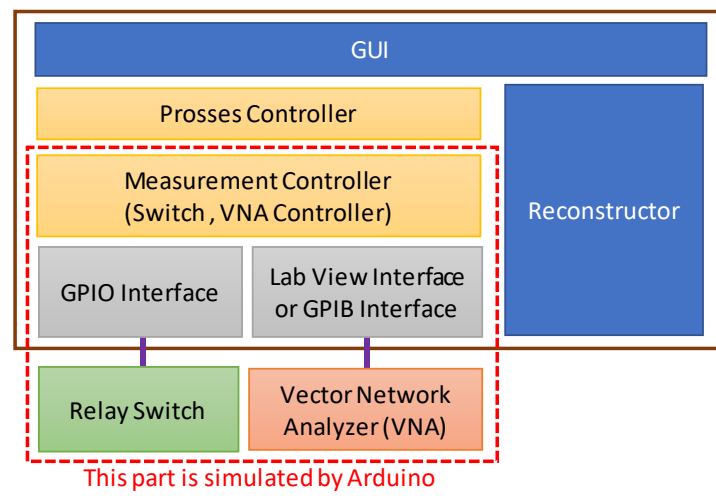

(b)

Figure 1 (a) Component diagram of the system; and (b) The framework's modules diagram

\subsection{Development of Microwave Image Reconstruction Using Low-Rank Compressive Sensing.}

\subsubsection{Input signal}

The input signal came from two kinds of measurement, measurement simulated with software and real measurement.
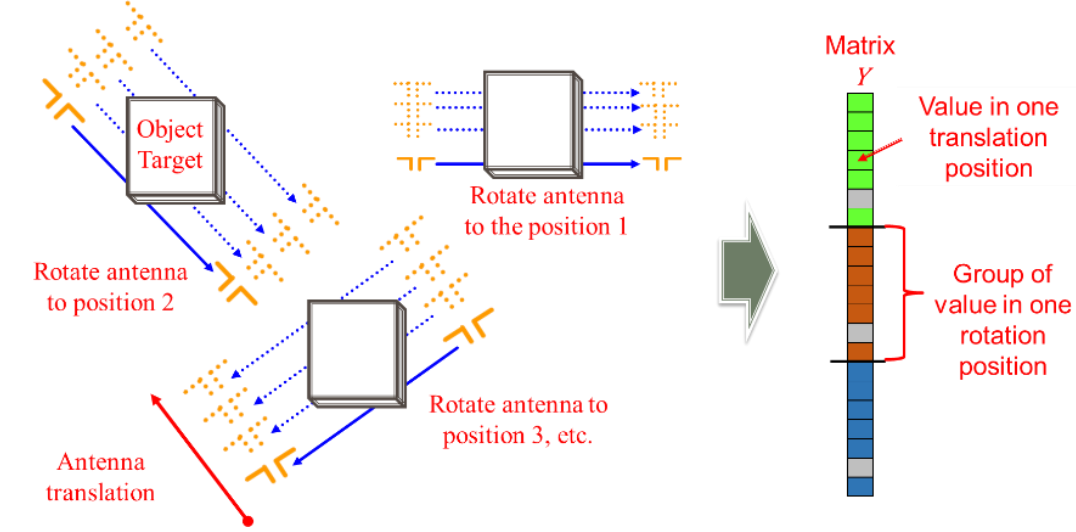

Figure 2 The mechanism of measurement and the matrix as the measurement result

The object being measured is a phantom, which represents a brain tumor. Phantoms are made in two-layer blocks where the outer layer represents brain tissue, and the inner layer represents tumor tissue with higher permittivity than brain tissue. The phantom and the simulation measurement were created and performed using CST software (for electromagnetic field simulation). Three $\mathrm{GHz}$ microwaves are passed into the phantom. Subsequently, the signal is captured and measured to be reconstructed as an image.

The measurement mechanism is shown in Figure 3. Data acquisition uses two dipole antennas opposite the object in the middle of them. Then, various translations and rotations of the two-dipole antennas were conducted to obtain a collection of measurement signal values to be reconstructed into an image. 
Previous research (Razzak et al., 2019) has shown that a combination of forty translations and three rotations gives the best reconstruction results of measurement data from software simulation. Therefore, we use these data to test qualitatively and quantitatively. Furthermore, we compare them with previous research results. Whereas for real measurement, the data are only to be compared to previous research results, whether it can improve reconstruction results or not. Hence, data from a combination of twenty translations and three rotations are used.

\subsubsection{Sparse and low-rank compressive sensing algorithm (SLR-CS) solved with ADMM}

Compressive sensing is a reconstruction approach that measures fewer data samples, typically sparse in a particular domain, but containing the maximum amount of information. The advantage of compressive sensing is that it is an efficient algorithm concerning data usage (Qaisar et al., 2013). The compressive sensing algorithm can be represented as the following linear equation:

$$
Y=\Phi X+E
$$

where $Y \in \mathbb{R}^{L \times 1}$ is the measurement matrix and the captured signal after passing through the phantom. $\Phi \in \mathbb{R}^{L \times m}$ is a projection matrix that maps the matrix $X$ into the matrix $Y, X \in$. $\mathbb{R}^{m \times 1}$ is the signal to be reconstructed. $E \in \mathbb{R}^{L \times 1}$ is the error factor. This error factor can be influenced by noise or scanning errors. $L$ is the number of measured microwave signal data, and $m$ is the number of image pixels resulting from the reconstruction.

The $X$ must be sparse. Accordingly, to ensure this, $X$ can be represented as:

$$
X=\Psi \mathrm{W}
$$

where $\Psi \in \mathbb{R}^{m \times m}$ is a sparse dictionary - a matrix used as a domain to represent a signal that has been reconstructed to be sparse, so $\mathrm{x}$ can be ascertained sparse. Wavelet transform (WT), Fast Fourier Transform (FFT) and Discrete Cosine Transform (DCT) are used for the processing, projection and decomposition of the signal (Nusantara et al., 2016; Muntasa, 2017; Basari and Kurniawan, 2019). In this research, DCT is selected as the sparse dictionary. $W \in \mathbb{R}^{m \times 1}$ is a matrix to be found as a reconstruction of CS in the sparse domain. Equation 2 substitutes for Equation 1 to become the following equation:

$$
Y=\Phi \Psi W+E
$$

$\Phi \Psi$ is constant, and it can be substituted as $F$. Therefore, Equation 3 can be simplified as:

$$
Y=\mathrm{F} W+E
$$

where $F$ is a reconstruction matrix.

Equation 4 is then formulated as an optimization problem to minimize the number of non-zero elements using the $W$ matrix's $l_{1}$ norm to consider the sparse data. A constraint is that the error should be less than the error tolerance $E$. The optimization problem equation in the constrained problem is as follows:

$$
\min _{W}\|W\|_{1} \text { subject to }\|F W-Y\|_{2} \leq E
$$

Equation 5 is nonconvex and hard to solve. Correspondingly, to make it convex, it is formed as an unconstrained problem, and the equation becomes as follows:

$$
\min _{W} \frac{1}{2}\|F W-Y\|_{2}^{2}+\lambda\|W\|_{1}
$$

Equation 6 minimizes the number of data terms. The first term aims to minimize the difference between the $F W$ reconstruction and the measurement data $Y$ to obtain the most accurate reconstruction results. The second term aims to minimize the number of non-zero $W$ matrix elements to guarantee the $W$ matrix's sparse properties, where the sparse properties are computationally close to the $l_{1}$ norm.

SLR-CS is implemented by adding nuclear norm regularization to Equation 6, then solved by the alternating direct method of multipliers (ADMM) method. Therefore, it is formulated with the following equation: 


$$
\min _{W} \frac{1}{2}\|F W-Y\|_{F}^{2}+\lambda_{l 1}\|W\|_{1}+\lambda_{n n}\|W\|_{*}
$$

where $\|W\|_{*}$ is a nuclear norm regularization. Moreover, $\lambda_{l 1}$ and $\lambda_{n n}$ are regularization parameters for the sparse $\left(I_{1}\right)$ and nuclear norm $(n n)$.

Then, Equation 7 is rewritten into a formulation equivalent to a constraint. Next, it is processed into the alternating direction method of multipliers (ADMM) algorithm. We utilize the auxiliary matrix variables $V_{1}, V_{2}$ and $V_{3}$. The formulation is as follows.

$$
\begin{gathered}
\min _{V_{1}, V_{2}, V_{3}, V_{4}}\left\{\frac{1}{2}\left\|V_{1}-Y\right\|_{F}^{2}+\lambda_{l 1}\left\|V_{2}\right\|_{1}+\lambda_{n n}\left\|V_{3}\right\|_{*}\right\} \\
\text { s.t } V_{1}-F W=0, V_{2}-W=0, V_{3}-W=0
\end{gathered}
$$

Let

$$
V=\left[\begin{array}{l}
V_{1} \\
V_{2} \\
V_{3}
\end{array}\right], G=\left[\begin{array}{l}
F \\
I \\
I
\end{array}\right], B=\left[\begin{array}{ccc}
-I & 0 & 0 \\
0 & -I & 0 \\
0 & 0 & -I
\end{array}\right]
$$

The augmented Lagrangian function is optimized concerning $W, V$ and $D$, as follows:

$$
\mathcal{L}(W, V, D)=\frac{1}{2}\left\|V_{1}-Y\right\|_{F}^{2}+\lambda_{l 1}\left\|V_{2}\right\|_{1}+\lambda_{n n}\left\|V_{3}\right\|_{*}+\frac{\mu}{2}\|G W+B V-D\|_{F}^{2}
$$

where $\mu>O$ is a positive penalty parameter, $D=\left[D_{1}^{T} D_{2}^{T} D_{3}^{T}\right]^{T}$ and $D_{i}=\frac{1}{\mu} M_{i}$, with $\mathrm{i}=1, . ., 3$ and $M_{i}$ containing the scaled Lagrange multipliers.

The ADMM proceeds by minimizing $\mathcal{L}(W, V, D)$ iteratively and sequentially. To elaborate on the ADMM steps, the optimization concerning $W, V_{1}, V_{2}, V_{3}$ and $\mathrm{D}$ is shown in Figure 3. $X$ results from image reconstruction.

\subsubsection{Implementation and evaluation}

We tuned the regularization parameters and iterations number to obtain the best reconstruction result. The image reconstruction result is analyzed qualitatively and quantitatively. Quantitative analysis uses the mean square error (MSE) and structural similarity index (SSIM) parameters. The more similar and the smaller the error of the reconstructed image to the reference image/expected image, the better the reconstruction method. We compared the reconstruction results with other algorithms like SART, sparse CS and CS with total variation (TV) (Razzak et al., 2019). In qualitative analysis, the reconstructed images are compared based on visual appearance and brightness, contrasts and shape.

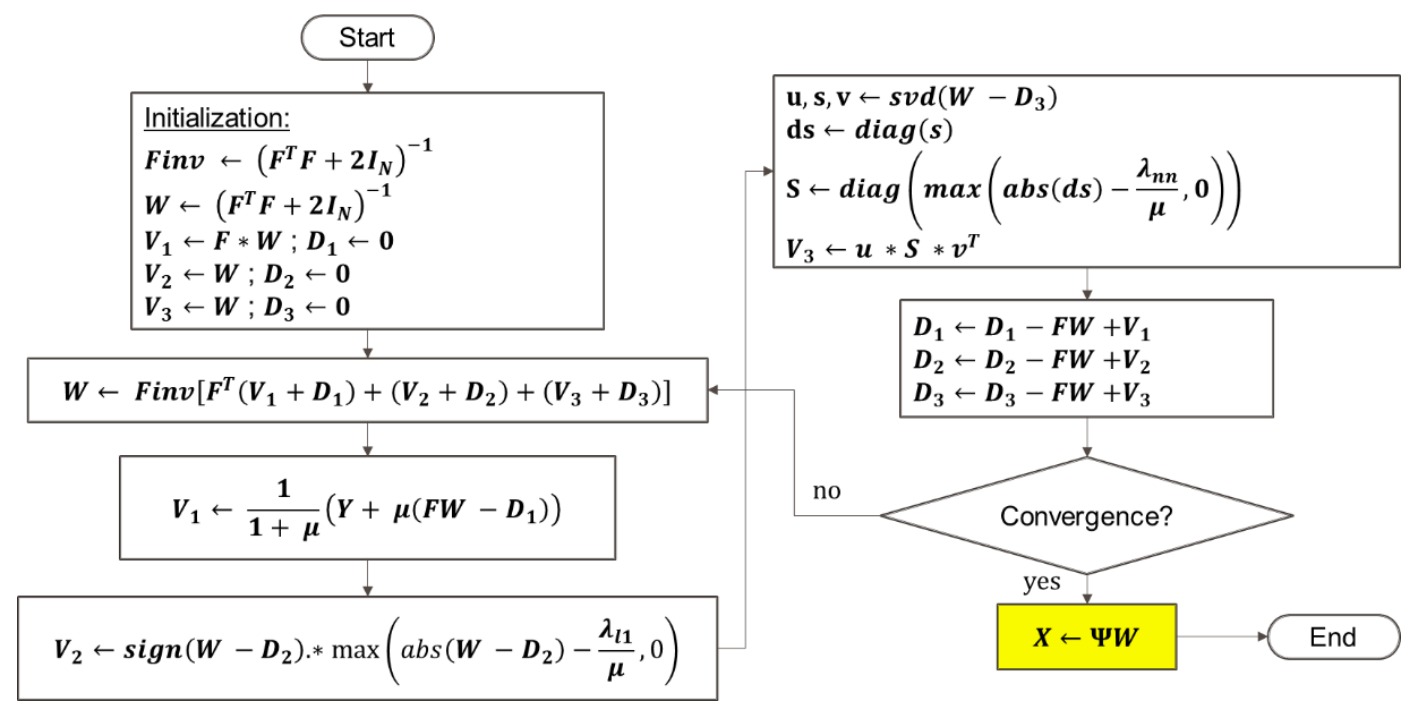

Figure 3 The image reconstruction flow chart 


\section{Results and Discussion}

The scope of this research is limited to developing a framework with a novel reconstruction algorithm inside. Hence, instead of system accuracy, performance evaluation is measured using framework functionality and algorithm performance. The system accuracy will be possible to ascertain once the framework functionality has been tested using Raspberry Pi connected with real VNA and switches. Furthermore, the system can be deployed and tested as a product of a portable brain tumor detector.

\subsection{Framework Functionality}

Figure 4a shows a prototype microwave-based brain tumor detection system consisting of a framework (controller and image reconstructor) running on the Raspberry Pi platform and hardware for data acquisition (VNA, Switch and Antenna), which are currently being simulated with Arduino. In Arduino, the microwave measurement value is stored, which is then accessed by the framework by giving commands for Arduino to send measurement data.

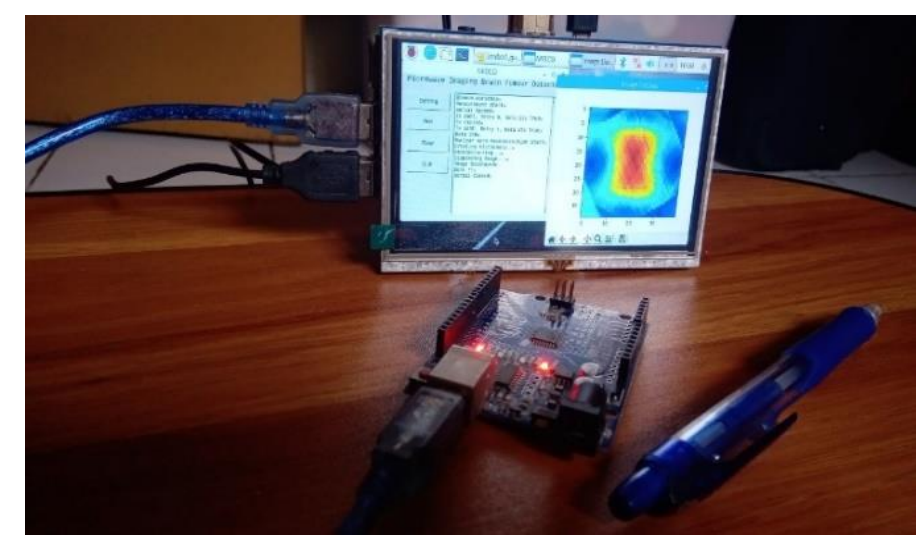

(a)

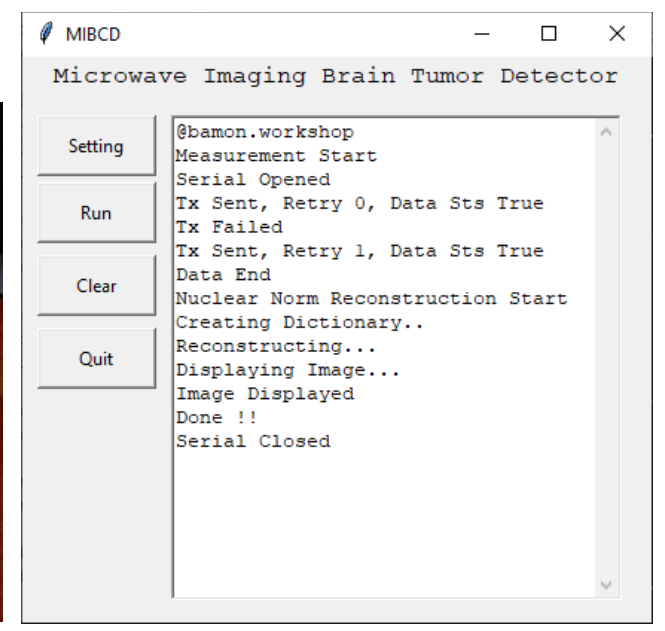

(b)

Figure 4 (a) The controller and image reconstructor prototype; and (b) The framework's GUI

The main view of the framework is shown in Figure 4b. A button "Setting" sets the image reconstruction parameters, a button "Run" performs measurement execution and image reconstruction, and a text box displays a log of running processes or error messages. Process logs range from serial communication access to measurement data acquisition, dictionary creation, reconstruction process and image display.

The evaluation of the framework is carried out using the software evaluation standard. The checkpoints are window display, setting saving, all button function, device communication, image reconstruction process, image display and error handling. The evaluation shows the framework can function as expected.

\subsection{Microwave Image Reconstruction Performance}

For the measurement data from CST, the qualitative result is shown in Figure 5. Compared with the previous reconstruction algorithm (CS with total variation), SLR-CS gives the same result of reconstructed image size and heat map color that distinguish tumors and tissues. The quantitative results are shown in Table 1. SLR-CS can increase similarity and decrease errors shown by the SSIM and MSE parameters, respectively.

The regularization parameter applied is determined by looking for the best combination of $\mu, \lambda_{I 1}$ and $\lambda_{n n}$ to produce the maximum SSIM value and minimum MSE. The determination process is performed automatically in the code. The best results are obtained 
at $\mu=0.0001, \lambda_{11}=0.05$ and $\lambda_{n n}=0.005$. The number of iterations to minimize matrix $\mathrm{W}$ was also determined by looking for the maximum SSIM and minimum MSE values. The experiment shows that $10 \times$ iterations produce the best value.

We evaluated the algorithm using available real measurement data. SLR-CS still gives the same image reconstruction result compared with CS added with TV regularization. The quantitative result in Table 2 shows that low-rank compressive sensing gives the same result with CS added with the TV algorithm.

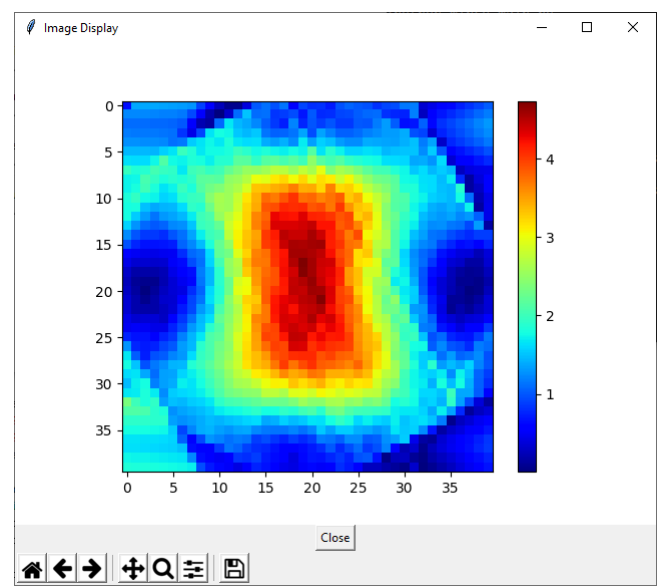

(a)

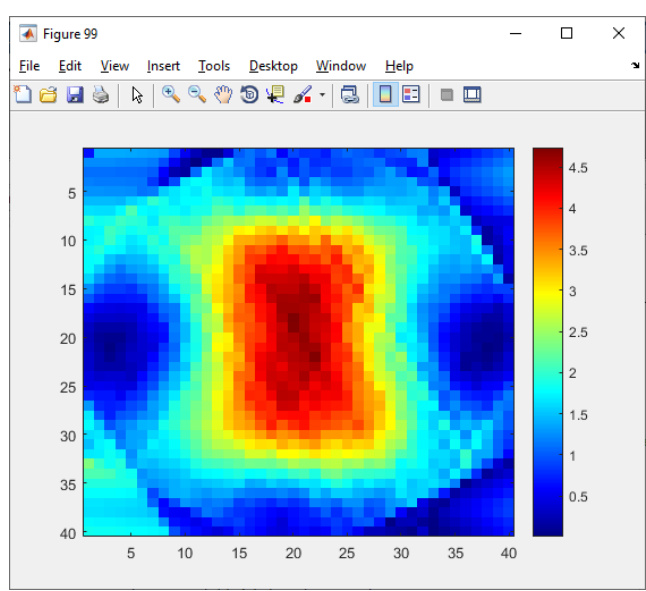

(b)

Figure 5 The reconstructed image by: (a) low-rank compressive sensing algorithm, written in Python; and (b) CS with total variation, written in MATLAB

Table 1 Comparison of quantitative parameters on simulated measurement

\begin{tabular}{ccc}
\hline Algorithm & SSIM & MSE \\
\hline SART & 0.4248 & 0.2019 \\
Sparse CS & 0.2728 & 0.2344 \\
CS + TV & 0.4427 & 0.1900 \\
SLR-CS & 0.4550 & 0.1844 \\
\hline
\end{tabular}

Table 2 Comparison of quantitative parameters on real measurement

\begin{tabular}{ccc}
\hline Algorithm & SSIM & MSE \\
\hline CS + TV & 0.00108 & 0.77 \\
SLR-CS & 0.00107 & 0.76 \\
\hline
\end{tabular}

The reconstruction result of this real phantom has a very high error because the input measurement signal is taken by a combination of twenty translations and three antenna rotations. Meanwhile, as mentioned in Section 2.2.1, the experimental results in previous research (Razzak et al., 2019) demonstrate the best results occur in a combination of forty translations and three antenna rotations.

\subsection{Future Works}

The CS was added with the TV method (Razzak et al., 2019), and the SLR-CS method was applied to the signal measurement shown in Figure 1. The microwaves are captured straight between a transmitter antenna and a receiver antenna after passing the object. Microwave measurement used array antennas with CS and the TV reconstruction algorithm applied (Guo et al., 2017), in which signals are passed through the object and scattered and reflected by it. Therefore, the SLR-CS method can also be applied to reconstruct an image 
from signals captured by array antennas. However, it needs further investigation related to the projection matrix and sparse library.

The framework development can be continued by adding VNA and switches controls, and the Raspberry Pi is connected to the real switches and antennas. Furthermore, it can make and mass produce the finished product of a microwave-based portable brain tumor detector.

\section{Conclusions}

This research has successfully designed and implemented a framework for controller and image reconstructor components of a universal, open-source, multi-platform, portable microwave-based brain tumor detector. The framework has been implemented in the Python language using Python libraries that support scientific computing. It can run on Raspberry $\mathrm{Pi}$, a card-sized computer platform. Frameworks can be operated in the simulation mode as appropriate for the user and can function according to predetermined specifications.

Algorithm development shows that low rankness by nuclear norm can be applied as regularization in microwave image reconstruction under the compressive sensing (CS) approach. Compared to the CS method added with TV, which has given the best results compared to the SART and FBP methods, the proposed method can give the same results and perhaps even better ones.

Qualitatively, the proposed SLR-CS algorithm shows the same image reconstruction in color and size to differentiate tumor and tissue. Quantitatively, this method can provide a better similarity and error value to the reference image, measured by SSIM and MSE parameters. The SLR-CS method provides a 45\% similarity rate (SSIM) and an 18\% pixel error rate (MSE), which is a 1\% increase from previous studies (CS added with TV).

\section{Acknowledgments}

The authors acknowledge Universitas Indonesia's support through the Q3 Research Grant 2020 and Q1Q2 Research Grant 2019 under contract number NKB-0309/UN2.R3.1/HKP.05.00/2019.

\section{References}

Ali, M.A., Moghaddam M., 2010. 3D Nonlinear Super-Resolution Microwave Inversion Technique using Time-Domain Data. IEEE Transactions on Antennas and Propagation, Volume 58(7), pp. 2327-2336

Aprilliyani, R., Prabowo, R.G., Basari., 2017. Comparison Analysis between SART and ART Algorithm for Microwave Imaging. PhotonIcs \& Electromagnetics Research Symposium - Fall (PIERS - FALL),

Basari., Ramdani, S., 2019. Evaluation on Compressive Sensing-based Image Reconstruction Method for Microwave Imaging. PhotonIcs \& Electromagnetics Research Symposium Spring (PIERS-Spring)

Chandra, R., Zhou, H., Balasingham, I., Narayanan, R.M., 2015. On the Opportunities and Challenges in Microwave Medical Sensing and Imaging. IEEE Transactions on Biomedical Engineering, Volume 62(7), pp. 1667-1682

Copper Mountain Technologies, 2020. Vector Network Analyzer Automation, Available Online at: https://coppermountaintech.com/automation/, Accessed on May 20, 2020 
Dilman, I., Akinci, M.N., Cayoren, M., Akduman, I., 2017. Differential Microwave Imaging of the Stroke-affected Brain via Diffraction Tomograph. The 25th Telecommunications ForUm TELFOR

Donoho, D.L., 2006. Compressed Sensing. IEEE Transactions on Information, Volume 52(4), pp. 1289-1306

Elevani, D., Ramadhan, R., Parastika, D.T., Basari., 2016. Evaluation of Algebraic Reconstruction Technique Algorithm for Microwave Imaging. Electromagnetic Research Symposium (PIERS), Shanghai

Elevani, D., Ramadhan, R., Parastika, D.T., Basari., Sanpanich, A., 2017. On the Performance of Algebraic Reconstruction Technique Algorithm for Microwave Imaging. IEEE $5^{\text {th }}$ Asia-Pacific Conference on Antennas and Propagation (APCAP), Taiwan

Gao, H., Jiang, X., 2013. Process on the Diagnosis and Evaluation of Brain Tumors. Cancer Imaging, Volume 13(4), pp. 466-481

Guo L., Bialkowski K., Abbosh A., 2017. Compressive Sensing using Approximated Total Variant Transform to Reduce the Required Number of Antennas in Radar-Based Medical Imaging. IEEE International Symposium on Antennas and Propagation \& USNC/URSI National Radio Science Meeting, USA

Giampouras, P.V., Themelis, K.E., Rontogiannis, A.A., Koutroumbas, K.D., 2016. Simultaneously Sparse and Low-Rank Abundance Matrix Estimation for Hyperspectral Image Unmixing. IEEE Transaction on Geoscience and Remote Sensing

Hugeng, H., Kurniawan, R., 2016. Development of the 'Healthcor' System as a Cardiac Disorders Symptoms Detector using an Expert System based on Arduino Uno. International Journal of Technology, Volume 7(1), pp. 78-87

Luo, L., Yang, J., Qian, J., Yang, J., 2014. Nuclear Norm Regularized Sparse Coding. 22 ${ }^{\text {nd }}$ International Conference on Pattern Recognition, pp. 1834-1839

Muntasa, A., 2017. Windowing System Facial Detection based on Gabor Kernel Filter, Fast Fourier Transform, and Probabilistic Learning Vector Quantization. International Journal of Technology, Volume 8(1), pp. 196-208

Nusantara, A.C., Purwanti, E., Soelistiono, S., 2016. Classification of Digital Mammogram based on Nearest-Neighbor Method for Breast Cancer Detection. International Journal of Technology. Volume, 7(1), pp. 71-77

Qaisar, S., Bilal, R.M., Iqbal, W., Naureen, M., Lee, S., 2013. Compressive Sensing: From Theory to Applications, a Survey. Journal of Communications and Network, Volume 15(5), pp. 443-456

Ramadhan, R., Prabowo, R.G., Aprilliyani, R., Basari., 2017. Comparison Analysis between Filtered Back Projection and Algebraic Reconstruction Technique on Microwave Imaging. International Symposium on Biomedical Engineering (ISBE), Bali, Indonesia

Ramdani, S., Astyani, A.P., Basari., 2018. Filtered Back Projection and Simultaneous Algebraic Reconstruction Technique for Image Formation on Square-shaped Physical Phantom Aimed at Microwave Imaging Applications. PhotonIcs \& Electromagnetics Research Symposium (PIERS-Toyama)

Ranson, R.G., 2015. Measurement Automation using Python. ARMMS RF \& Microwave Society

Raspberry Pi Foundation, 2020. Raspberry Pi 3 Model B+. Available Online at: https://www.raspberrypi.org/products/raspberry-pi-3-model-b-plus/, Accessed on April 3, 2020

Razzak, I.H., Rizkinia, M., Basari., 2019. Image Reconstruction based on Compressive Sensing using Total Variation Spatial Regularization for Microwave Imaging. Photonics \& Electromagnetics Research Symposium, Rome, Italy 
Shtoda, D.O., Arkusha, Y.V., Mustetsov, M.P., 2017. The Microwave Imaging Method for Express Diagnostic of Cancer. IEEE First Ukraine Conference on Electrical and Computer Engineering (UKRCON), Ukraine

Ulas, C., 2016. Spatio-temporal MRI Reconstruction by Enforcing Local and Global Regularity via Dynamic Total Variation and Nuclear Norm Minimization. IEEE $13^{\text {th }}$ International Symposium on Biomedical Imaging (ISBI), Prague, Czech Republic

World Health Organization (WHO), 2018. Cancer. Available Online at: https://www.who.int/news-room/fact-sheets/detail/cancer, Accessed on April 3, 2020

Yang, K., 2019. Nonlocal Weighted Nuclear Norm Minimization Based Sparse-Sampling CT Image Reconstruction. IEEE $16^{\text {th }}$ International Symposium on Biomedical Imaging (ISBI)

Yang, W., 2017. A Nuclear Norm-based Matrix Regression-based Projections Method for Feature Extraction. IEEE Access, Volume 6 